\title{
Wave-packet propagation study of the charge-transfer dynamics of Rydberg atoms with metal surfaces
}

\author{
E. So, M. T. Bell, and T. P. Softley \\ Department of Chemistry, University of Oxford, Chemistry Research Laboratory, Oxford OX1 3TA, United Kingdom
}

(Received 23 June 2008; published 12 January 2009)

\begin{abstract}
A wave-packet propagation study is presented of the ionization dynamics of xenon and hydrogen Rydberg atoms interacting with a metal surface in the presence of an external field. The calculations are performed using a Coulomb-wave discrete variable representation, which allows an efficient extension of previous calculations to a higher principal quantum number. The wave-packet calculations include nonadiabatic effects at avoided energy level crossings. Ionization probabilities as a function of distance from the surface are compared with complex-scaling calculations, which assume purely adiabatic traversal of the avoided crossings. A comparison is made between the dynamics calculated for the "normal" experimental situation, where the applied field is oriented so as to repel positive ions away from the surface, versus the dynamics for the reversed field situation, in which electrons are repelled from the surface. Overall it is clear that reversing the field direction has a pronounced effect on the ionization dynamics for any given starting level and that the nonadiabatic effects are most pronounced in the reversed field case. For certain field ranges, electron flux is found to be "backscattered" away from the surface in the reversed field configuration. Preliminary mean-field calculations are also presented to evaluate the effect of the acceleration of the atom on the ionization dynamics.
\end{abstract}

DOI: 10.1103/PhysRevA.79.012901 PACS number(s): 34.35.+a, 32.80.Ee, 34.70.+e, 34.50.Gb

\section{INTRODUCTION}

The interaction of Rydberg atoms and molecules with metallic surfaces, and the associated resonant charge-transfer process, has been the subject of many previous experimental [1-7] and theoretical studies [8-19]. As is discussed in more detail in our previous work [4,5], such studies are of relevance to the understanding of plasma-surface interactions, ion sputtering, nanoscale pattern deposition, the study of electronically excited states at surfaces, and the thermal emission of Rydberg atoms from a surface, and they also offer possibilities for control of certain forms of surface chemistry.

As a Rydberg atom or molecule approaches a metal surface, the energies of the Rydberg states are perturbed by the surface potential generated by the image charges of the Rydberg electron and core. At small atom-surface separation ( $\approx 4 n^{2} a_{0}$, where $n$ is the principal quantum number), the Rydberg atom or molecule may be ionized by resonant charge transfer of the Rydberg electron from the discrete localized atomic or molecular level to the continuum of delocalized unoccupied metal states, with which the Rydberg electron is degenerate in energy. Experimentally, this surface ionization can be observed by detecting the resulting positively charged ion through the introduction of a large positive potential to the metal surface to act as an extraction field [4-7]. If no ion extraction field is applied or the field is reversed, the positively charged ion will be accelerated (by its image charge) towards the metal surface. A number of competing processes control the fate of the ion [20], such as collisional dissociation (for a Rydberg molecule), resonant neutralization, and Auger neutralization. If an adlayer is present on the surface then ion-molecule surface chemistry is also possible. The multielectron Auger process involves the emission of secondary electrons from the metal surface; in principle, these electrons may be detected by simply reversing the direction of the applied field relative to that used in the "normal" ionextraction scheme. Indeed, in recent experiments involving the interaction of $\mathrm{H}_{2}$ Rydberg molecules with metal surfaces, we have succeeded in detecting an electron signal from the surface ionization [21], and therefore an understanding of the ionization dynamics in the reversed field is required. Although it is not possible to deal with the full dimensionality of a hydrogen molecule-surface system at present (the rotational motion of the ion core playing an important role [4,5]), the focus on atomic systems, both hydrogenic and nonhydrogenic, reveals dynamical properties that should be characteristic of all Rydberg systems.

For experiments in which the ions are detected, the results are typically presented in the form of a "surface-ionization profile," in which the total ion signal is plotted as a function of extraction field, and this profile can be directly related to the probabilities of ionization at different distances from the metal surface [4]. Recent time-dependent wave-packet propagation studies [16,22] have shown that for a Rydberg system with many electronic states closely spaced in energy, the inclusion of nonadiabatic effects is essential if one wants to accurately reproduce the experimental surface-ionization profiles, since the coupling between these Rydberg states has large effects in the appearance of the profile. The advantage in using such a time-dependent approach is that all the nonadiabatic transitions that may occur between the Rydberg states during the collisional process can be taken into account naturally. This time-dependent wave-packet propagation approach has been used by Sjakste et al. to investigate the charge transfer of a xenon Rydberg atom at a metal surface [16], providing an explanation for the experimental results of Hill et al. [6]. In that particular theoretical study, the Rydberg states considered have lower principal quantum numbers $(n)$ than those studied experimentally $(7 \leqslant n \leqslant 8$ instead of $13 \leqslant n \leqslant 20$ ), and the collision velocities used in the calculations are up to two orders of magnitude larger than 
those used in the experiments. The difficulty in performing calculations at principal quantum numbers and velocities appropriate to experiments arises from the scaling properties of the size of the Rydberg orbital and the distance for the onset of surface ionization [9], both of which increase as $n^{2}$. As a result, on moving to higher quantum numbers the calculations rapidly become more computationally demanding, since the size of the grid upon which the wave packet is propagated, the number of grid points required to adequately describe the ionization process, and the number of time steps required to propagate the wave function before the Rydberg atom is surface ionized increases rapidly.

In the wave-packet calculations of Sjakste et al. [16] a change of variable in the cylindrical coordinates is performed to concentrate more grid points near the origin, in order to treat the Coulomb potential most efficiently. In this paper, we show that the resonant charge-transfer process can be more efficiently described using a Coulomb-wave discrete variable representation (CWDVR) [23-25] for the radial coordinates in the wave-packet calculations. This allows for studies involving both higher principal quantum numbers and/or lower velocities. The aim of the present work is to utilize the CWDVR in our time-dependent wave-packet propagation calculations alongside the time-independent complex-scaling approach [8] to gain further understanding on the surface-ionization dynamics of both hydrogenic and nonhydrogenic systems under various field conditions (ionand electron-extraction fields) and collisional velocities.

Full reviews of the CWDVR used in our wave-packet propagation, and of the regularized Lagrange-Laguerre DVR used in both the initial diagonalization of the Hamiltonian in the wave-packet calculation and in our complex-scaling calculations, are given in Refs. [23,26], respectively; only a brief summary will be given in this paper. Unless stated otherwise, we use atomic units throughout.

\section{THEORY AND IMPLEMENTATION}

To study the charge-transfer process of the Rydberg electron to a metal surface, we treat the center-of-mass motion of the ion core of the Rydberg atom classically and monitor the time evolution of the electronic wave function under the influence of the potential due to the metal surface. The initial quantum-state-selected electronic wave function is discretized on a spatial grid (see Section II B). To minimize the area covered by the numerical grid, and thus the total number of grid points, we have chosen to define the electronic coordinates with respect to the moving atomic frame. In the atomic frame, the ion core is taken to be stationary and is located at the origin, whilst the metal surface is incoming from the negative $z$ axis at a constant collisional velocity. This constant velocity assumption, which does not correctly describe the coupling between the nuclear and electron dynamics, is used as a first approximation. However, at the experimental range of collisional energies [4,6], this approximation cannot be fully justified and the errors associated with this assumption are discussed in Sec. III C. In performing the Galilean transformation from the surface-fixed frame, we have used an unmodified form of the metal surface po- tential. This is justified by the fact that the collisional velocities considered in this work are very much lower than the velocities of the metal electrons at the Fermi level [27]. The metal surface is assumed to be invariant with respect to rotation about the surface normal, and so the system exhibits cylindrical symmetry. With this assumption, the surfaceionization problem becomes two dimensional (see Fig. 2), and the azimuthal $\phi$ coordinate can be ignored. The evolution of the electronic wave function (in the two-dimensional $z-\rho$ plane) as the surface approaches is described by the timedependent Schrödinger equation (see Sec. II A), and is studied by applying the time-evolution operator to an initially selected wave function (see Sec. II C). The ionization probability of the Rydberg atom is then measured by calculating the outgoing electron flux into the metal surface and towards the edge of the spatial grid (see Sec. II E).

\section{A. Nuclear trajectory and the electron Hamiltonian}

As stated above, as a first approximation, the motion of the Rydberg ionic core is assumed to be classical and follows a straight line trajectory perpendicular to the surface, i.e., the atom-surface separation $d$ at time $t$ is given by

$$
d(t)=d_{0}-v_{\perp} t
$$

where $d_{0}$ is the initial atom-surface separation at $t=0$ and $v_{\perp}$ is the velocity of the Rydberg atom perpendicular to the surface.

The electronic wave functions are the solutions to the time-dependent Schrödinger equation as follows:

$$
i \frac{\partial}{\partial t} \Psi(\mathbf{r} ; t)=\hat{H} \Psi(\mathbf{r} ; t),
$$

with

$$
\hat{H}(\mathbf{r} ; t)=\hat{T}(r)+\hat{V}_{\text {atom }}(\mathbf{r})+\hat{V}^{\prime}(\mathbf{r} ; t) .
$$

In this equation, $\hat{T}(r)$ is the radial kinetic-energy operator, $\hat{V}_{\text {atom }}(\mathbf{r})$ is the "atomic" potential energy operator that includes the centrifugal potential (which has been expanded over a spherical basis in the equation below) and the Coulomb potential,

$$
\hat{V}_{\text {atom }}(\mathbf{r})=\sum_{l, m} \frac{l(l+1)}{2 \mu r^{2}}|l m\rangle\langle\operatorname{lm}|+\hat{V}_{c}(r),
$$

and $\hat{V}^{\prime}(\mathbf{r} ; t)$ is the "perturbing" potential-energy operator composed of the applied electric field potential $\hat{V}_{F}(z)$ and the cylindrically symmetric surface potential $\hat{V}_{\text {surf }}(z, \rho ; t)$,

$$
\hat{V}^{\prime}(\mathbf{r} ; t)=\hat{V}_{F}(z)+\hat{V}_{\text {surf }}(z, \rho ; t)=F z+\hat{V}_{\text {surf }}(z, \rho ; t) .
$$

The surface potential experienced by the Rydberg electron includes the attractive electron-image-electron interaction $V_{e e}$ and the repulsive electron-image-proton interaction $V_{e p}$,

$$
V_{\text {surf }}(z, \rho ; t)=V_{e e}(z ; t)+V_{e p}(z, \rho ; t) .
$$

For the electron-image-electron interaction we adopt the analytical Jellium-model potential proposed by Jennings et al. [28] for a free-metal surface 


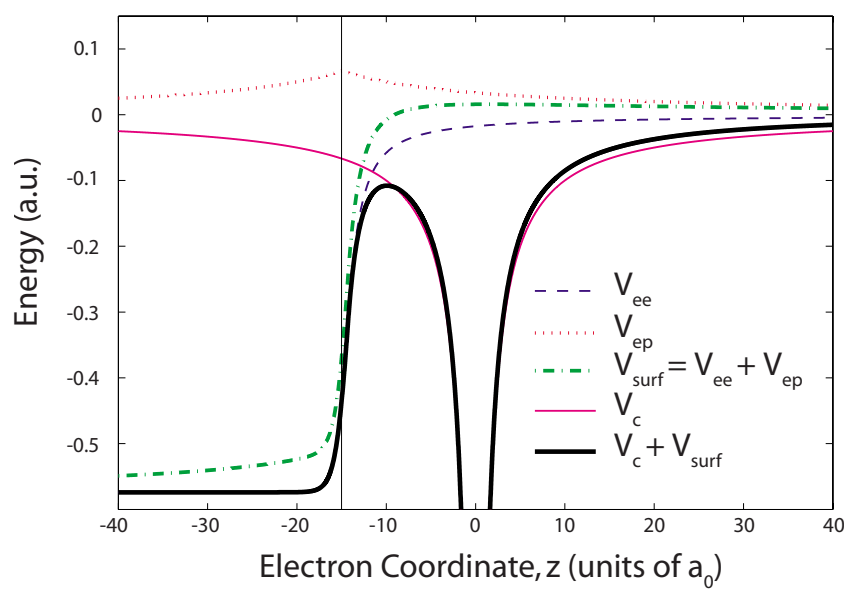

FIG. 1. (Color online) Plot of the surface interactions $V_{e e}$ and $V_{e p}\left[\right.$ Eqs. (7) and (8)], the total surface potential $V_{\text {surf }}$, the Coulomb potential $V_{c}$, and the total potential experienced by a Rydberg electron in the absence of an external electric field $\left(V_{c}+V_{\text {surf }}\right)$. The surface is positioned at the electron coordinate (with respect to the ion-core position) of $z=-15 a_{0}$.

$$
V_{e e}=\left\{\begin{array}{cl}
\frac{-1+\exp \left\{-\lambda\left[z+d(t)-z_{0}\right]\right\}}{4\left(z+d(t)-z_{0}\right)} & \text { if }[z+d(t)]>z_{0} \\
\frac{V_{0}}{A \exp \left\{B\left[z+d(t)-z_{0}\right]\right\}+1} & \text { otherwise, }
\end{array}\right.
$$

with

$$
A=-1-4 V_{0} / \lambda, \quad B=-2 V_{0} / A .
$$

For the aluminum surface studied here, the parameters above take the values $V_{0}=-0.574, z_{0}=0.7$, and $\lambda=1.25$ [28]. The electron-image-proton potential is treated classically using the image-charge model and is given by

$$
V_{e p}=\frac{1}{\sqrt{[d(t)+|z+d(t)|]^{2}+\rho^{2}}} .
$$

The form of the total potential, $V_{\text {surf, }}$ and its contributions $\left(V_{e e}\right.$ and $\left.V_{e p}\right)$ are shown in Fig. 1.

The attractive interaction between the ion and the image ion is ignored here since it will lower the energy of all states equally and does not affect the wave function of the Rydberg electron [though it is included in our later calculations (see Sec. III C)]. Note that due to the cylindrical symmetry of the overall potential, the magnetic quantum number $m_{l}$ is conserved; only $m_{l}=0$ states are considered for all cases studied in this paper.

The initial wave function is found by diagonalizing the Hamiltonian given by Eq. (3) at time $t=0$ and distance $d$ $=d_{0}$. The initial atom-surface separation $d_{0}$ is chosen to be large enough that negligible surface ionization takes place before the atom reaches this separation, and that the surface potential is not yet large enough to bring about any level crossings. For the calculations carried out in this paper, $d_{0}$ is typically set to values $\geqslant 6 n^{2} a_{0}$, where $n$ is the principal quantum number of the state of interest. In this initial diagonal-

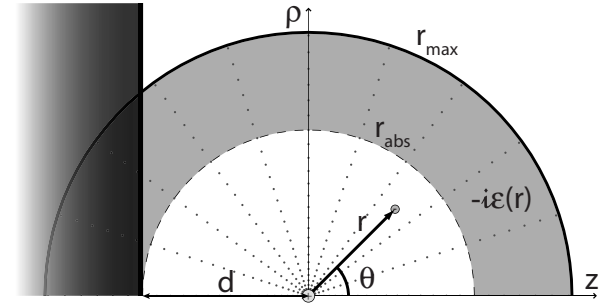

FIG. 2. A schematic illustration of the CWDVR grid, as used in the wave-packet calculations, in the reference frame of the ion core. Note the higher concentration of grid points near the origin and the regularly spaced points at large radius. The distance of the ion core from the metal surface is labeled $d, r_{\text {abs }}$ is the lower bound of the absorbing potential and the position of the radial flux plane, and $r_{\max }$ is the maximum radius of the numerical grid and the upper bound of the absorbing potential.

ization step, the angular coordinate is described by a Legendre polynomial in the finite basis representation (FBR) [29]. Although it is possible to carry out the diagonalization directly in the CWDVR basis for the radial co-ordinate (see below), at such large separations from the surface where the surface ionization is negligible and the radial extension of the wave function into the metal surface is small, it is unnecessary to include such a large number of grid points at large radii. We therefore carry out the initial diagonalization step using a regularized Lagrange-Laguerre basis [26] for the radial coordinate, and then project the appropriate initial wave function onto the CWDVR basis.

\section{B. Coulomb-wave discrete variable representation}

The Coulomb-wave discrete variable representation (CWDVR) proposed by Dunseath et al. [23] is used in our calculations to describe the electron wave function in the radial coordinate. The CWDVR consists of grid points defined as the positive roots of a positive-energy Coulomb function. The grid points are nonuniformly distributed, with a high concentration of points near the Coulomb singularity, and an almost regular spacing at large distances (see Fig. 2). The particular advantage of using this discrete variable representation (DVR) is that the Coulomb singularity is treated naturally and so no regularization of the potential near the origin is required. The distribution of grid points can be controlled by adjusting two grid parameters $\kappa$ and $Z$ as defined in Ref. [23]. The grid parameter $Z$ varies the concentration of points near the Coulomb singularity, while $\kappa$ varies the separation between grid points at large distances. Thus, the CWDVR is particularly suited for the surface-ionization problem, which involves wave functions that are bound by the Coulomb potential at small distances, but become free waves when the electron is transferred into the metal. Typical calculations for the $n=10$ states of the hydrogen atom and the $\nu=7$ states of the xenon atom ( $\nu$ is the effective principal quantum number) presented in this work involved 603 and 474 radial points, respectively.

In the CWDVR, the kinetic-energy matrix is given by a simple analytical expression [23], and the potential-energy matrix in the radial coordinate is diagonal, with matrix ele- 
ments given by the value of the potential at the grid points as follows:

$$
\begin{gathered}
T_{i j}^{D V R}=-\delta_{i j} \frac{W\left(r_{i}\right)}{6}+\left(1-\delta_{i j}\right) \frac{1}{\left(r_{i}-r_{j}\right)^{2}}, \\
V_{i j}^{D V R}=\delta_{i j} V\left(r_{i}\right),
\end{gathered}
$$

where $W\left(r_{i}\right)=\left(\kappa^{2}+2 Z / r_{i}\right)$, and $\kappa$ and $Z$ are the grid parameters. In our computation, the positive-energy Coulomb wave function and its derivative (required for calculating the quadrature weights) for the CWDVR are computed using the COUFLG subroutine of Barnett [30], and the roots, which are the positions of the radial grid points, are found using the Newton-Raphson method [31].

\section{Time propagation}

The formal solution of Eq. (2) is

$$
\Psi(t+\Delta t)=e^{-i \hat{H} \Delta t} \Psi(t)
$$

Starting from an initial wave function $\Psi(t)$, the wave functions of the system at a later time, $\Psi(t+\Delta t)$, may be computed through the action of the propagator $e^{-i \hat{H} \Delta t}$. For small time steps, the propagator may be approximated by the symmetric split operator [32]

$$
e^{-i(\mathbf{H}-i \epsilon) \Delta t}=e^{-i\left(\mathbf{V}^{\prime} / 2\right) \Delta t} e^{-i\left(\mathbf{T}+\mathbf{V}_{\text {atom }}-i \epsilon\right) \Delta t} e^{-i\left(\mathbf{V}^{\prime} / 2\right) \Delta t},
$$

where $\mathbf{T}, \mathbf{V}_{\text {atom }}$, and $\mathbf{V}^{\prime}$ are the matrix representations of the operators defined in Eq. (3), and $-i \epsilon(r)$ is the absorbing potential (see Sec. II D). The error associated with the split operator expression [Eq. (12)] decreases as the size of the time steps decreases, and, for typical calculations carried out in this work, a time step of $\Delta t=1$ a.u. is found to be sufficient. From Eq. (12), the advantage of using a radial DVR and an angular FBR-DVR [29] is now clear. The kineticenergy matrix $\mathbf{T}$ in the CWDVR is given by simple analytical expressions [Eq. (9)] and the time-independent potentialenergy matrix $\mathbf{V}_{\text {atom }}$ is diagonal in the radial DVR and the angular FBR, and is given by the value of the potential at the radial grid points for a given angular-momentum quantum number $l$. Hence the middle term in Eq. (12) can be precomputed before the propagation of the wave function.

The time-dependent potential term $\mathbf{V}^{\prime}$, which depends on both the radial and angular coordinates is diagonal in both the radial DVR and angular DVR. Since the Legendre polynomial FBR-DVR transformation matrix can be easily computed by diagonalizing a tridiagonal matrix [33], the operation of $e^{-i\left(\mathbf{V}^{\prime} / 2\right) \Delta t}$ on the wave function can be evaluated by simply transforming the wave function between the angular FBR and DVR and multiplying by a diagonal matrix; the matrix elements are given by the exponential of the value of the potential at the given radial and angular points,

$$
e^{-i\left(\mathbf{V}^{\prime} / 2\right) \Delta t}=\mathbf{U} \mathbf{D}_{\mathbf{V}}^{\mathbf{e}} \mathbf{U}^{\dagger}
$$

where $\mathbf{D}_{\mathbf{V}}^{\mathbf{e}}$ is a diagonal matrix with elements $D_{V i i}^{e}=e^{V_{i i}^{\prime D V R}}$, and $\mathbf{U}$ is the angular FBR-DVR transformation matrix [29].

\section{Absorbing potential}

To remove the spurious reflections of the wave function at the edge of the grid and to maintain the correct outgoingwave boundary condition for the electron wave function, the Manolopoulos transmission-free absorbing potential $[34,35]$ $-i \epsilon(r)$ is added to the Hamiltonian.

$$
\mathbf{H}=\mathbf{H}-i \boldsymbol{\epsilon}(r),
$$

where $-i \epsilon$ is a diagonal matrix with elements

$$
-i \epsilon(r) h\left(r-r_{\mathrm{abs}}\right) \text {. }
$$

$r$ is the electron radial coordinate, $r_{\mathrm{abs}}$ is the radial lower bound of the absorbing region, and $h(f(r))$ is the Heaviside step function. The negative complex potential $-i \epsilon(r)$ has the form

$$
-i \epsilon(r)=-i \frac{y(x)}{2}\left(\frac{2 \pi}{\Delta r}\right)^{2}
$$

where

$$
x=\frac{c\left(r-r_{\mathrm{abs}}\right)}{\Delta r},
$$

and the approximate form of $y(x)$ is [35]

$$
y(x)=\frac{4}{(c-x)^{2}}+\frac{4}{(c+x)^{2}}-\frac{8}{c^{2}} .
$$

Here $\Delta r=r_{\max }-r_{\mathrm{abs}}$ is the width of the absorbing region, $r_{\max }$ is the maximum radius for the numerical grid, and the numerical value of $c \approx 2.62206[34,35]$. The width of the absorbing region, $\Delta r$, depends on the momentum of the outgoing wave. For the calculations carried out in this paper, it has been found that a width of $30 a_{0}$ is sufficient to remove the outgoing wave function at the edge of the grid. A schematic illustration of the CWDVR grid, the absorbing boundary, and the coordinates used in the wave-packet calculations is shown in Fig. 2.

\section{E. Ionization detection}

To study the ionization of the Rydberg electron, the total electron density lying within the region enclosed by the lower bound of the absorbing region $\left(r_{\mathrm{abs}}\right)$ and the metal surface is monitored as a function of the atom-surface distance. The derivative of the electron population inside this "bound" region with respect to the atom-surface separation may be interpreted as the probability (per unit length) of the Rydberg atom ionizing at that distance. This is equivalent to having a stationary circular flux plane with radius $r_{\mathrm{abs}}$ that is truncated by a moving (z-) flux plane, which represents the approach of the metal surface. It is important to include the "vacuum" boundary as well as the metal flux plane because, on carrying out the wave-packet propagation calculations presented below, it was found that not all of the electron density escapes into the metal; under certain field conditions, some density escapes through the vacuum side away from the metal. It is also important to have a sufficiently large radius $r_{\text {abs }}$ such that the Rydberg electron may be considered 
TABLE I. The coefficients $A_{l}$ used in this work for the pseudopotential of the xenon atom $[14,16]$.

\begin{tabular}{cccccc}
\hline \hline & $l=0$ & $l=1$ & $l=2$ & $l=3$ & $l>3$ \\
\hline$A_{l}$ & -1.17984 & -0.89700 & -0.49906 & -0.00823 & 0 \\
\hline \hline
\end{tabular}

as ionized beyond this radial limit. For the calculations carried out in this paper, $r_{\mathrm{abs}}$ is typically set to values of $\geqslant 6 n^{2} a_{0}$. To study the fraction of "forward" $\left(\theta>90^{\circ}\right)$ and "backward" $\left(\theta<90^{\circ}\right)$ flux, forward and backward radial flux planes using the flux operator [36] are also used to study the outgoing electron density.

\section{F. Xenon pseudopotential}

For a nonhydrogenic atom, the interaction of the excited Rydberg electron with the ion core is a complex many-body problem and cannot be solved exactly. A simple way to treat this interaction is to use a set of $l$-dependent pseudopotentials, which can be adjusted such that the calculated atomic energies of the Rydberg states using the pseudopotentials match the experimentally determined quantum defects $[37,38]$, whilst maintaining the correct number of radial nodes. Following previous studies $[14,16]$, a Bardsley pseudopotential [39] of the form

$$
V^{P S}(r)=-\frac{1}{r}+\sum_{l} V_{l}(r)|l\rangle\langle l|,
$$

with

$$
V_{l}(r)=A_{l} e^{-B r^{2}}-\frac{\alpha_{d}}{2\left(r^{2}+d^{2}\right)^{2}}-\frac{\alpha_{q}}{2\left(r^{2}+d^{2}\right)^{3}}
$$

is applied to the case of the xenon atom, where $\alpha_{d}$ $=4.044$ a.u., $\alpha_{q}=14.235$ a.u., $B=0.01$, and $d$ is set to $1.0 a_{0}$ [14].

For the $n=7$ states, the coefficients $A_{l}$ used in this work are given in Table I. Note that these parameters are different from those given in Ref. [16]. The parameters used in this work have been adjusted to produce radial wave functions with the correct number of radial nodes as well as energies corresponding to the experimental quantum defects.

\section{G. Time-independent complex-scaling calculations}

The effect of the surface potential on the energy levels and the energy widths $(\Gamma)$ associated with the finite lifetimes of these Stark states is determined using the timeindependent complex-scaling approach, as previously described by Nordlander and co-workers [8]. In the present work parabolic coordinates [40] are used in the implementation of the formalism; this choice of coordinates is justified by the cylindrical symmetry of both the surface potential and the externally applied electric field. Test results were compared with previous calculations in spherical coordinates for the $\mathrm{H}$ [9] and Xe [14] atoms to ensure correct application of the method. Similar to conventional matrix diagonalization methods, where the energies of bound states are given by the eigenvalue of a Hermitian Hamiltonian matrix, the complexscaling approach involves the diagonalization of a "complexrotated" non-Hermitian matrix. This complex rotation imposes the correct resonance boundary condition on the wave function of the resonance states, and the energies and halfwidths $\Gamma / 2$, related to the lifetimes $\tau$, of decaying states by $\Gamma=\frac{1}{\tau}$, are obtained from the real and imaginary parts of the eigenvalues, respectively.

In the complex-scaling calculations, the instantaneous population $n(Z=d)$ at a given atom-surface separation $d$ (i.e., the fraction of atoms not ionized by the time the atom reaches the distance $d$ ), is derived for a specific quantum state by

$$
n(d)=\exp \left(\frac{1}{v_{\perp}} \int_{\infty}^{d}-\Gamma(Z) d Z\right),
$$

where $\Gamma(Z)$ is the $Z$-dependent level width (e.g., as plotted in Fig. 5). The probability of ionization at a given distance $P(Z=d)$ for a given collisional velocity can then be calculated by taking the derivative of Eq. (21) with respect to the atom-surface separation. It should be noted that this calculation implicitly requires an assumption that the system follows a given level adiabatically (see below for further discussion).

\section{RESULTS AND DISCUSSION}

The wave-packet propagation and complex-scaling calculations are applied in this section to collisions of $\mathrm{H}$ and $\mathrm{Xe}$ Rydberg atoms with an aluminum surface. Particular attention is given to the effects of reversing the direction of the externally applied electric field on the ionization dynamics of the Rydberg atom. This reversed field case has been neglected in previous theoretical work, presumably because of the lack of related experimental data. For both directions of the field, the effects of the magnitude of the applied field and the collisional velocity of the Rydberg atom are examined.

The output of the wave-packet propagation calculations are presented below in Figs. 3, 7, 8, 10, 13, and 16 as plots of the ionization probability (per unit length) $P(Z)$ versus distance of the atom from the surface $Z$ for specified initial quantum state, field, and collisional velocity. For the cases where the direction of the field is set for ion extraction $(F$ positive), these ionization curves can be related to experimental ionization profiles [4-7] [ion extraction probability $S(F)$ versus field $(F)]$ by the integration

$$
S(F)=\int_{\infty}^{Z_{\min }(F)} P(Z) d Z .
$$

$Z_{\min }(F)$ is the minimum position of ionization for which the given applied field can pull the bare ion away from the surface [5].

\section{A. Surface ionization of the hydrogen Rydberg atom}

\section{Comparision of the effects of the ion- and electron-extraction fields on the ionization dynamics}

Figure 3(a) presents the wave-packet propagation results for the $n=10, m_{l}=0$ states of the hydrogen atom approaching 


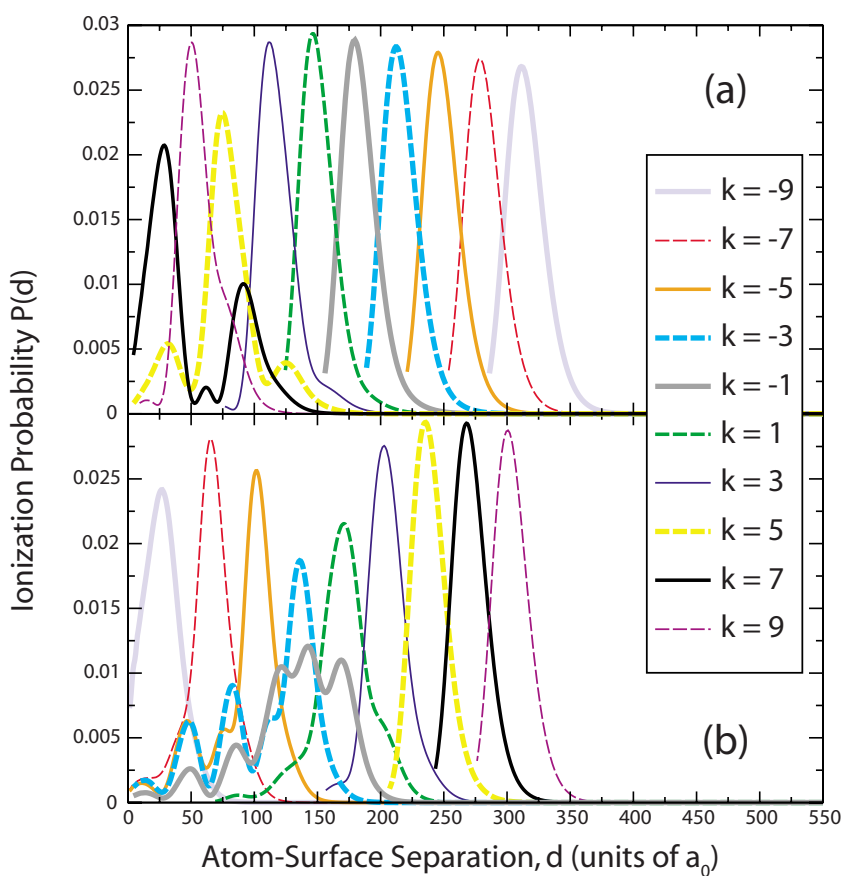

FIG. 3. (Color online) Wave-packet propagation results for the ionization of the $n=10, m_{l}=0$ states of the hydrogen atom approaching a metal surface under an externally applied field of (a) $+1 \times 10^{-6}$ a.u. $\equiv 5.14 \times 10^{5} \mathrm{~V} \mathrm{~m}^{-1}$ (ion-extraction field) and (b) -1 $\times 10^{-6}$ a.u. (electron-extraction field). The collisional velocity of the Rydberg atom is $1.4 \times 10^{-3}$ a.u. $\equiv 3.06 \times 10^{3} \mathrm{~m} \mathrm{~s}^{-1}$.

an aluminum surface with a collisional velocity of 1.4 $\times 10^{-3}$ a.u. $\left(\equiv 3.06 \times 10^{3} \mathrm{~ms}^{-1}\right)$ and under a constant ionextraction field of $F=1 \times 10^{-6}$ a.u. $\left(\equiv 5.14 \times 10^{5} \mathrm{~V} \mathrm{~m}^{-1}\right)$. Figure 3(b) presents the results obtained when the direction of this extraction field is reversed, i.e., an electron-extraction field of $-1 \times 10^{-6}$ a.u. is applied. A field of magnitude 1 $\times 10^{-6}$ a.u. is relatively weak for this principal quantum number, i.e., well below the Inglis-Teller field [41] (for $n$ $=10, F_{I-T}=3.3 \times 10^{-6}$ a.u.). The initially prepared Rydberg Stark states are labeled in Fig. 3 by the parabolic quantum number $k$, which runs from $-(n-1-|m|)$ to $(n-1-|m|)$ in steps of two [41]. As shown in Fig. 4, the positive- $k$ states are blueshifted in the presence of a field (of either positive or negative sign) whereas the negative- $k$ states are redshifted. Figure 3(a) shows that for the positive ion-extraction field, the redshifted negative- $k$ states are ionized at greatest distance from the surface. These states have wave functions with electron density that is initially (at large atom-surface separations) oriented towards the surface (see Fig. 4), hence they are most readily ionized. For the negative electronextraction field, however, it is the blueshifted positive- $k$ states that are ionized at greatest distance [see Fig. 3(b)], as it is these for which the electron density is initially oriented towards the surface.

This simplistic picture of wave-function orientation in the applied field appears to provide a qualitative explanation of the variation in ionization probabilities with $k$ quantum number and with field direction. But the wave functions and the energy levels are perturbed as the Rydberg atom approaches the surface, and this evolution must be considered to explain

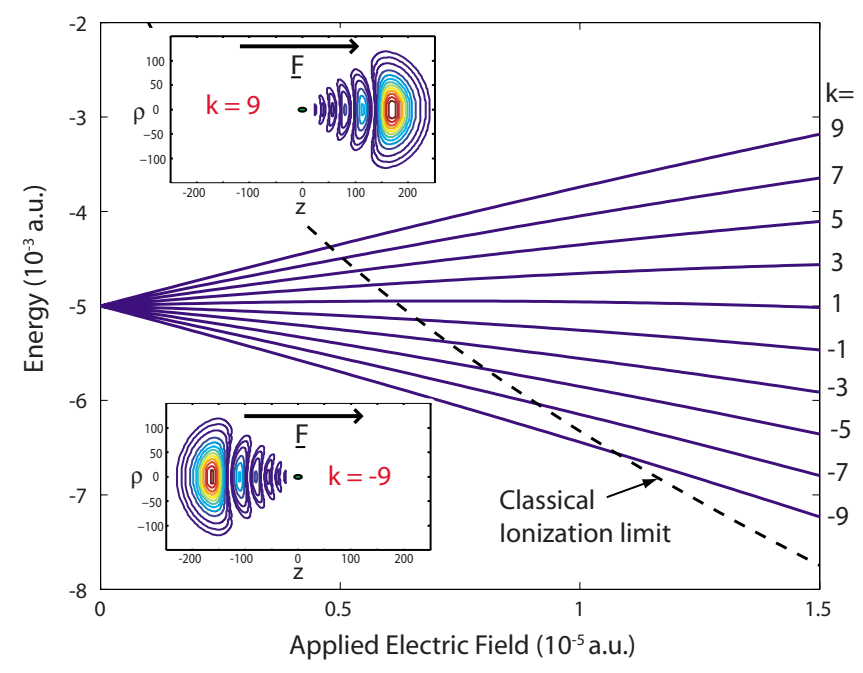

FIG. 4. (Color online) The energies of the hydrogen atom $n$ $=10, m_{l}=0$ states as a function of the strength of the applied electric field. $k$ is the parabolic quantum number. Insets: Plots of the electronic wave function of the extreme Stark states of the $n=10$ manifold, with parabolic numbers $k=9$ and $k=-9$, under the applied field strength of $1 \times 10^{-6}$ a.u. Note that the direction of the applied field is towards positive $z$ (i.e., away from the surface) $[1$ a.u. $=5.14$ $\times 10^{11} \mathrm{~V} \mathrm{~m}^{-1}$.

a number of other effects; for example, reversing the direction of the field leads to more oscillations in the ionization curves for the electron extraction case [Fig. 3(b)], and in this case the onset of ionization occurs at a shorter distance for the most easily ionized states.

Figure 5 shows the calculated energies and lifetimes for $n=10$ as a function of distance from the surface, obtained using the time-independent complex-scaling approach (see Sec. II G). Unsurprisingly, Fig. 5 confirms that generally, for a given atom-surface separation, the widths of surfaceoriented states are significantly larger than those of vacuumoriented states, i.e., surface-oriented states are surface ionized further away from the surface.

The different shifts in energies shown in Figs. 5(a) and 5(c) for the various states can be explained with reference to the form of the surface potential (Fig. 1). For a vacuumoriented state, mainly the long-range surface potential, which is dominated by the repulsive electron-image-proton interaction $V_{e p}$ is probed. The repulsive electron-image-proton interaction depends on both the electron $z$ coordinate and the atom-surface separation [Eq. (8)], and it increases as the atom-surface separation decreases. Thus, the energies of the vacuum-oriented Rydberg states increase with decreasing atom-surface separation. In contrast, for a surface-oriented state, the attractive surface potential may be probed provided that the atom-surface separation is sufficiently small. This attractive surface potential is dominated by the electronimage-electron potential $V_{e e}$, which lowers the energies of the Rydberg states. As the atom-surface separation decreases further, the electron-image-proton interaction, $V_{e p}$, becomes highly repulsive and once again dominates and the energies of the Rydberg states increase again.

For the positive field [Fig. 5(a)], the higher-energy states are initially vacuum oriented and there is therefore a spread- 


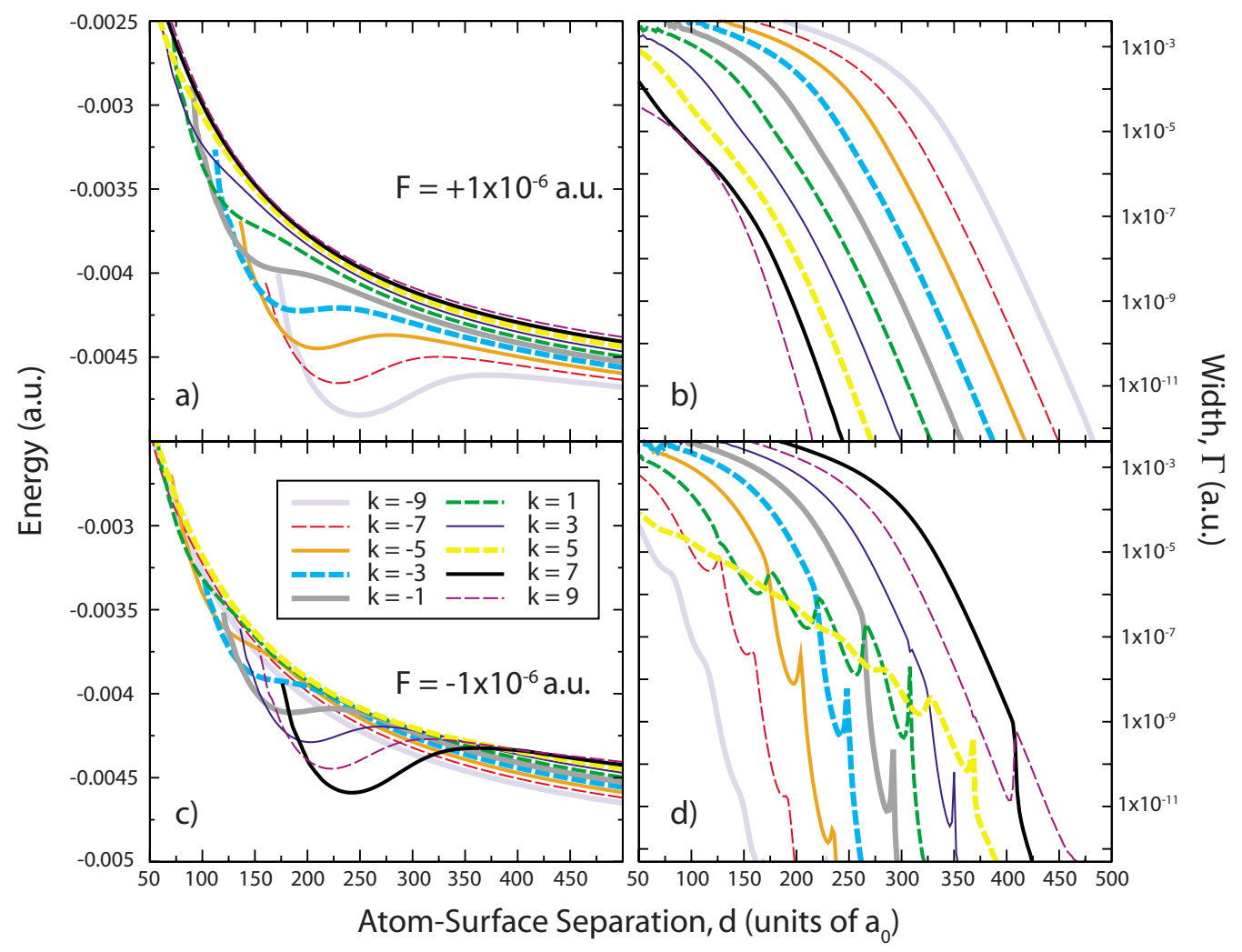

FIG. 5. (Color online) Energies (left panel) and widths (right panel) of the $n=10, m_{l}=0$ hydrogen atom Rydberg states under ion- and electron-extraction fields of $F=+1 \times 10^{-6}$ a.u. (top plots) and $F=-1 \times 10^{-6}$ a.u. (bottom plots) calculated using complex-scaling theory.

ing of the energy levels as the surface is approached, until short distances are reached where all states move up in energy. For the negative-field electron-extraction scheme, the higher-energy states are initially surface oriented in the field and will tend to lower their energy as the atom approaches the metal surface [Fig. 5(c)], while the lower-energy vacuum-oriented states rise in energy. Thus there is a tendency for the levels to converge and cross as the surface is approached. Unlike the case of a hydrogen atom in a homogeneous electric field (Fig. 4), avoided crossings occur in the presence of the image charge field even within the same $n$ manifold for both directions of field; the surface potential breaks the z-parity symmetry of the system allowing interaction between the levels.

The larger number of possible avoided level crossings that occur in the electron-extraction scheme is the origin of the greater tendency for oscillations in the ionization probability plots in Fig. 3(b) and the discontinuities in the plots of level widths in Fig. 5(d). The polarizations of the wave functions (vacuum or surface-oriented) may become mixed at the avoided crossings, changing the ionization dynamics. If the system follows the avoided crossings adiabatically then there will be sharp changes of ionization probability at distances corresponding to these crossings, reflecting sharp changes in the wave function.

The ionization curves are collectively shifted to lower atom-surface separations in Fig. 3(b) compared to Fig. 3(a). Figure 6 shows that, for an ion-extraction field ( $F$ positive), the potential barrier on the surface side of the ion core becomes lower and thinner, thus enhancing the surface- ionization process, while for the electron-extraction scheme ( $F$ negative) the potential barrier on the surface side of the core is raised and widened, thus hindering the surfaceionization process. It is also worth noting that for the electron-extraction field, the potential barrier on the vacuum side of the core is lowered, and so there is a nonzero probability of surface-induced ionization involving a "backscat-

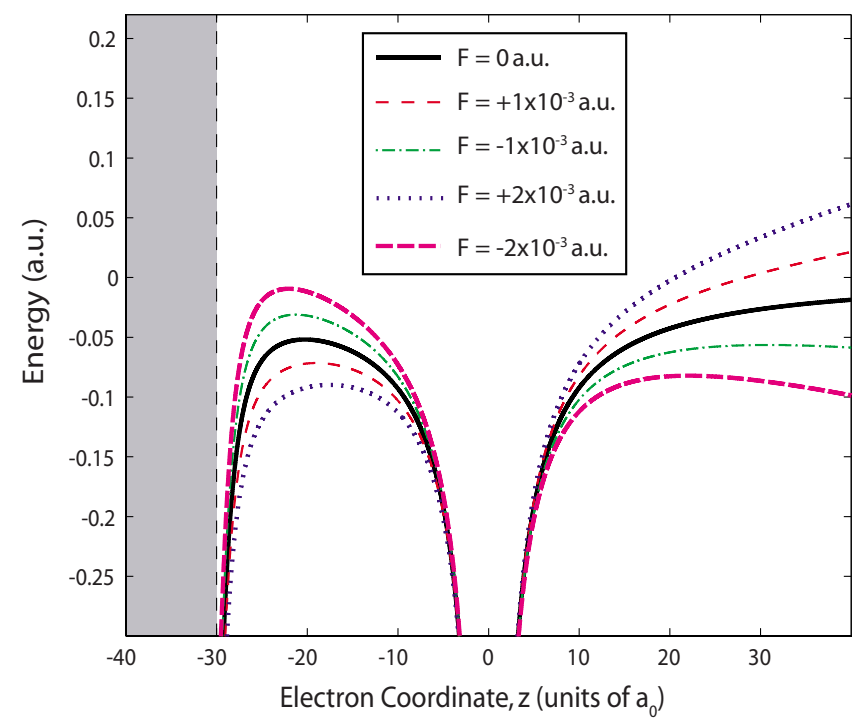

FIG. 6. (Color online) The total potential (sum of the Coulomb potential $V_{c}$, the surface potential $V_{\text {surf }}$, and the potential from the externally applied field $V_{F}$ ) experienced by the Rydberg electron at various electric fields. The metal surface is placed at $z=-30 a_{0}$. 


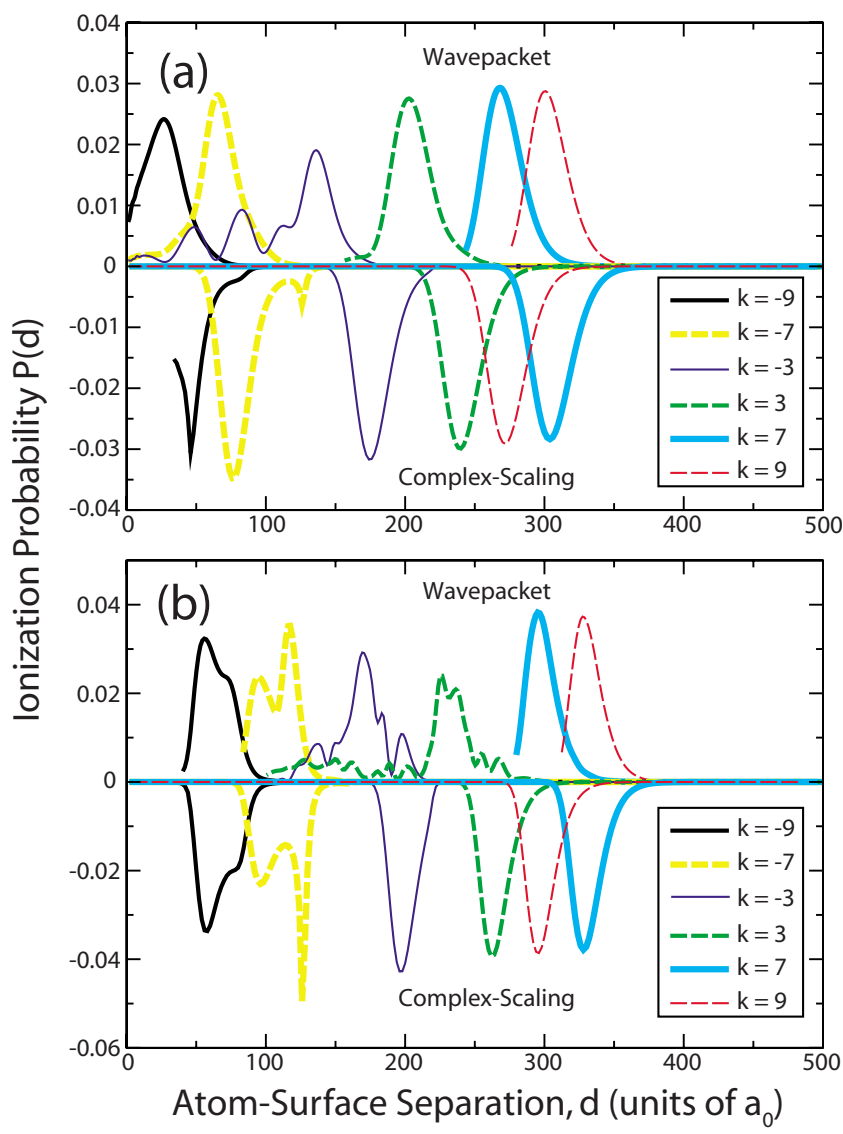

FIG. 7. (Color online) Comparison of the results from the wavepacket propagation calculations (positive axis) and the complexscaling calculations (negative axis) for the $n=10$ hydrogen Rydberg states approaching a metal surface with collisional velocity of (a) $1.4 \times 10^{-3}$ a.u. and (b) $1.4 \times 10^{-4}$ a.u., in the presence of an electron-extraction field of $-1 \times 10^{-6}$ a.u. Note that for clarity, only a selection of Stark states is presented here. It becomes increasingly difficult to obtain converged energies and widths from the complexscaling calculations at very low atom-surface separations $\left(<30 a_{0}\right)$, and so these results are not shown.

tered" flux of electrons (with respect to the nuclear trajectory) to occur (see Sec. III A 4).

\section{Effects of the collisional velocity: Adiabaticity and nonadiabaticity of crossings}

In the Landau-Zener avoided-crossing model, the extent to which crossings are traversed adiabatically is dependent on the gradient of the energy levels at the crossings, the collisional velocity of the Rydberg atom, and the magnitude of the energy gap [42]. Previous calculations based on complex-scaling theory have assumed that the avoided crossings are traversed adiabatically.

To examine the importance of nonadiabatic transitions as the Rydberg atom approaches the metal surface, Fig. 7 compares the ionization probability curves obtained under an adiabatic transition model from the complex-scaling calculations in Fig. 5, with those obtained from the time-dependent wave-packet calculations, which include all nonadiabatic effects. Results are shown for the hydrogen $n=10$ states in the

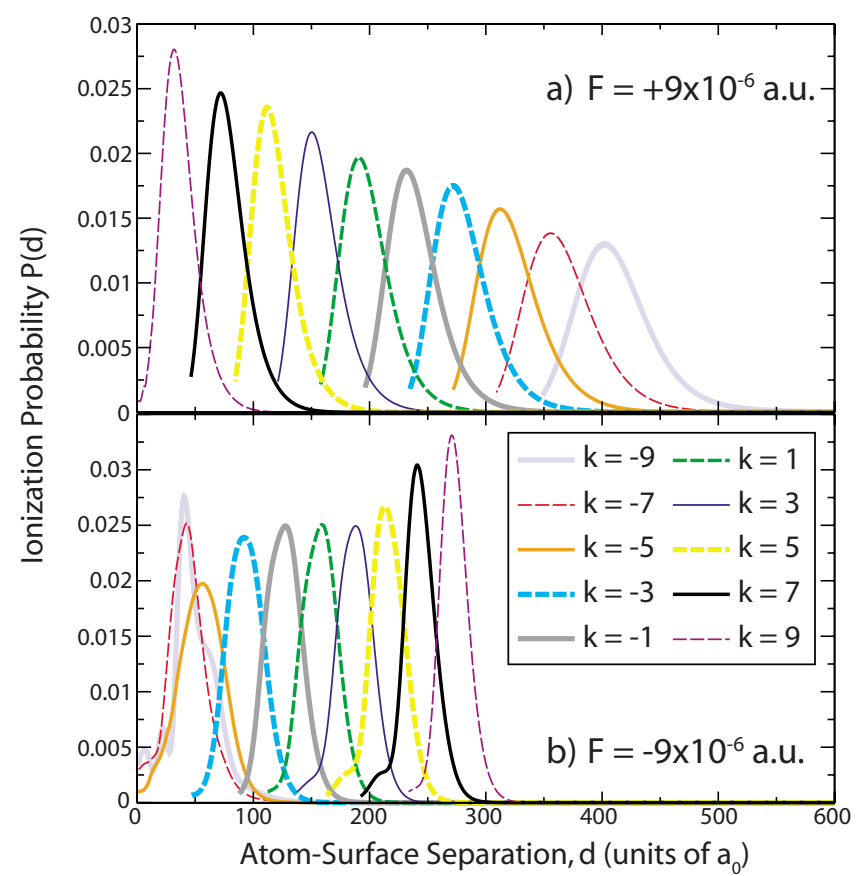

FIG. 8. (Color online) Wave-packet propagation results for the ionization of the $n=10, m_{l}=0$ states of the hydrogen atom approaching a metal surface with the collisional velocity of 1.4 $\times 10^{-3}$ a.u. and in the presence of an externally applied field of (a) $F=+9 \times 10^{-6}$ a.u. and (b) $F=-9 \times 10^{-6}$ a.u.

presence of an electron-extraction field $F=-1 \times 10^{-6}$ a.u. with collisional velocities of $1.4 \times 10^{-3}$ a.u. and 1.4 $\times 10^{-4}$ a.u. (the experimental velocity range typically is of the order of $1 \times 10^{-4}$ a.u. $\left.\equiv 219 \mathrm{~m} \mathrm{~s}^{-1}[4,5]\right)$. A "weak" electron-extraction field has been chosen because under these field conditions the Rydberg states will encounter a large number of avoided crossings as they approach the metal surface (see below). Significant differences are observed between wave-packet and complex-scaling results at both velocities, although as expected these are more pronounced at the highest velocities when a nonadiabatic passage through the crossings is more probable.

The positions of the ionization peaks in Fig. 7 are shifted to marginally lower atom-surface separations in the wavepacket results compared to the complex-scaling results, and this shift increases with the collisional velocity. The shifts in ionization peaks are attributable to the "time lag" between the ionization of the Rydberg atom and the detection of the Rydberg electron at the detection flux planes, and are dependent on the distance of ionization and the relative velocity of the ionized electron and the ion core. At typical experimental velocities of $\approx 1 \times 10^{-4}$ a.u. [4,5], it can be seen from Fig. 7 that this effect is very small ( $<1$ a.u.) and such a shift is acceptable for the present qualitative discussions.

\section{Effects of the magnitude of the applied electric field}

Figure 8 shows the wave-packet propagation results for the $n=10, m_{l}=0$ hydrogen Rydberg states for both positive and negative fields of magnitude $9 \times 10^{-6}$ a.u., and Fig. 9 shows the corresponding energies and widths calculated 


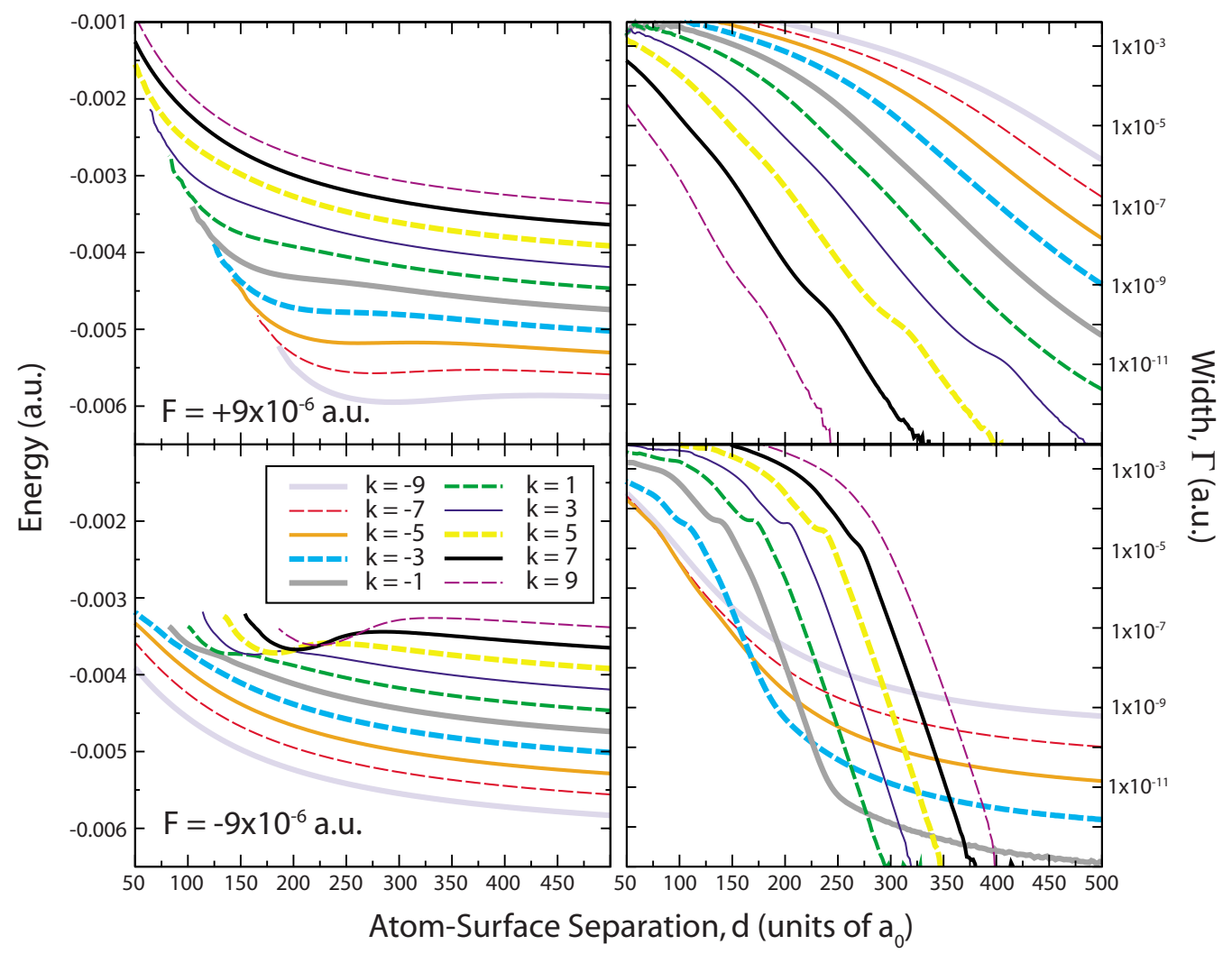

FIG. 9. (Color online) Energies and widths of the $n=10, m_{l}=0$ hydrogen Rydberg states under ion- and electron-extraction fields of $F$ $=+9 \times 10^{-6}$ a.u. (top plot) and $F=-9 \times 10^{-6}$ a.u. (bottom plot), respectively.

from complex scaling. By comparing Figs. 9 and 5 (calculated for $F=1 \times 10^{-6}$ a.u.) it can be seen that when the strength of the electric field is increased, the number of avoided level crossings decreases rapidly: the individual energy levels spread further apart at higher fields (Fig. 4), and the applied electric field competes with the field due to the surface image charges for distances where ionization occurs. In effect, an extra symmetry is imposed on the system by the strong field.

The comparison of Fig. 8(a) and Fig. 3(a) shows that for the ion-extraction scheme, both the lower-energy surfaceoriented states and the higher-energy vacuum-oriented states are ionized further away from the surface as the field is increased (this change is much smaller for the vacuum-oriented states). For the electron-extraction scheme [Fig. 8(b) versus Fig. 3(b)], the higher-energy surface-oriented states are ionized closer to the surface at higher fields, and the lowerenergy vacuum-oriented states are ionized further from the surface. As shown in Fig. 6 the lower potential barrier at higher fields facilitates ionization in the positive field and hinders ionization in the negative field. But in the latter case the vacuum-oriented states can also ionize via a backscattered flux, which is enhanced as the field increases (see below).

\section{Backscattering of the Rydberg electron}

Using the wave-packet propagation approach, the fraction of forward (towards the metal) and backward (away from the metal) electron flux can be calculated by detecting the flux going through a set of forward $\left(\theta>90^{\circ}\right)$ and backward $(\theta$ $<90^{\circ}$ ) radial flux planes positioned near the edge of the numerical grid.

The results for a field of $F=-9 \times 10^{-6}$ a.u. are shown in Fig. 10 (the backward flux has been plotted on the negative axis). As expected, only the most vacuum-oriented states

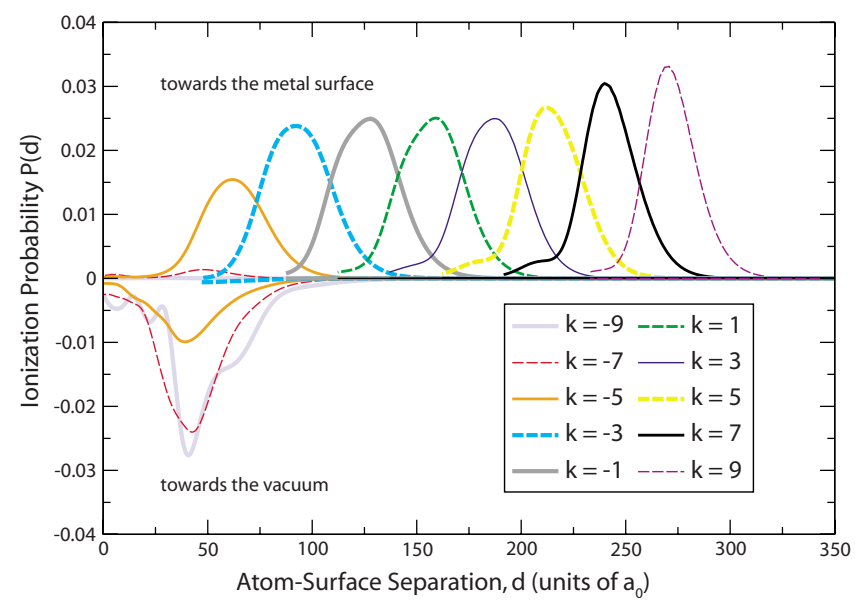

FIG. 10. (Color online) The electron flux measured by the forward $\left(\theta>90^{\circ}\right)$ and backward $\left(\theta<90^{\circ}\right)$ radial flux planes (positioned at $r_{\text {abs }}=930 a_{0}$ ) plotted as a function of atom-surface separation for the $n=10, m_{l}=0$ hydrogen states colliding with the surface with the collisional velocity of $1.4 \times 10^{-3}$ a.u. and in the presence of an electron-extraction field of $F=-9 \times 10^{-6}$ a.u. The flux measured by the backward flux plane is plotted on the negative axis. 
have a significant fraction of electron density escaping towards the vacuum side. It is also found that as the strength of the applied field increases, the fraction of backscattered electron flux increases, due to the further lowering of the vacuum-side potential barrier with the increasing field. However, the range of fields at which significant backscattered electron flux occurs is limited to the crossover region between surface ionization and direct field ionization, typically a field range $-1 / 20 n^{4}<F<-1 / 9 n^{4}$.

At higher electric-field strengths, direct-field ionization dominates and there would be a significant contribution to the width associated with this field ionization process, even at large separation from the surface where the surface potential is negligible. Figure 4 shows that for a field of \pm 9 $\times 10^{-6}$ a.u. the higher-energy states of the $n=10$ manifold lie above the classical ionization threshold. Nevertheless, the bulk of the electron density is located on the opposite side of the ion core to the saddle point created by the field potential, and therefore kinetically, the lowest-energy states with electron density located on the same side as the saddle-point field ionize first [41]. At a field of strength $-9 \times 10^{-6}$ a.u., the system has not fully undergone the transition from the pure surface-ionization regime to the pure field-ionization regime. The backscattered electron flux for the vacuum-oriented states is therefore still a surface-induced process, where the repulsive electron-image-proton potential $V_{e p}$ essentially "pushes" the electron over the potential barrier on the vacuum side of the ion core. Note however, the real experimental time scale between the application of the extraction field and surface ionization is of the order of microseconds $[4,5]$. Therefore the kinetic stability of higher-energy states in the Stark manifold (explained above) will have no effect, and in reality, the field range for the observation of backscattered electrons would be much smaller than suggested above.

\section{Summary of the hydrogen atom results}

For the hydrogen atom system, significant differences between the ion-extraction and electron-extraction fields are a consequence of the different orientation of the electron cloud relative to the surface, and of the greater number of energy level crossings as the surface is approached in the latter case. Even with this current constant velocity assumption, which neglects any ion-core acceleration, time-dependent wavepacket calculations are necessary to provide the most accurate ionization profiles because nonadiabatic effects can make a significant contribution to these profiles. A surfaceinduced electron flux is predicted to occur in the backward direction for the vacuum-oriented states at electronextraction fields close to the classical field ionization threshold.

\section{B. Surface ionization of the xenon Rydberg atom}

It is of interest to compare the above calculations with a study of the same effects in a nonhydrogenic system. Previous experimental studies [6] using xenon atoms have shown that the ionization dynamics of the xenon Rydberg atom under an ion-extraction field are very different from the hydrogenic predictions. The onset of ionization for the most

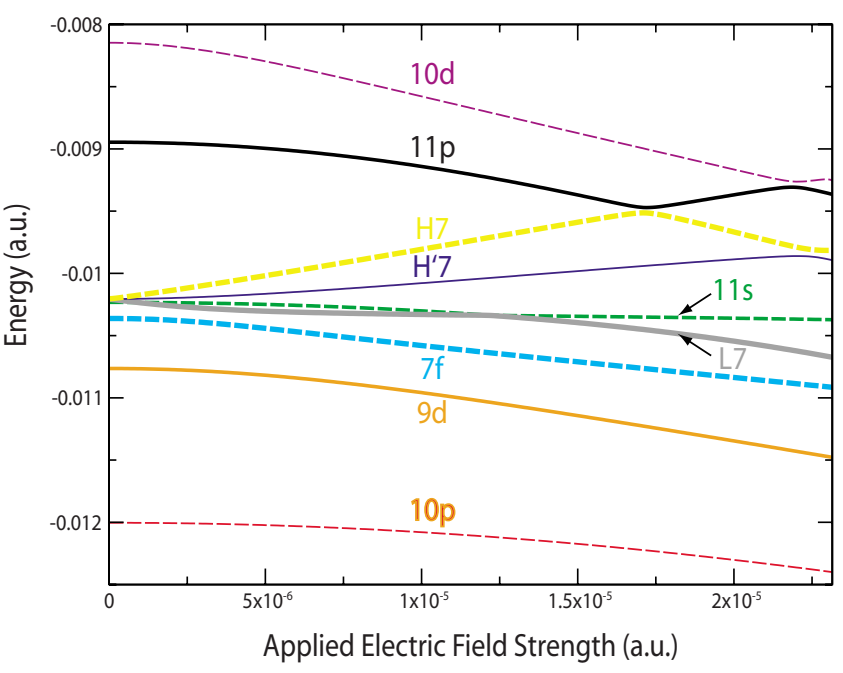

FIG. 11. (Color online) Stark map of the Rydberg xenon $\nu \approx 7$ states calculated using the Lagrange-Laguerre mesh method [26]. $\nu$ is the effective principal quantum number.

vacuum-oriented state in the manifold is very similar to that of the most surface-oriented state; this has been explained by the exchange of ionization behaviors of the Rydberg states near an avoided crossing between states of different $n$ on the Stark map [16]. The choice of xenon allows a comparison of our implementation of the wave-packet approach (using the CWDVR) directly with the implementation by Sjakste et al. [16], and potentially with the results of experiments [6].

In our calculations, the Coulomb potential $V_{c}$ in the Hamiltonian given by Eq. (3) is replaced by the pseudopotential $V^{P S}(r)$, given by Eq. (19). The Stark map (plot of the energy levels against the applied electric-field strength) for the $\nu \approx 7$ states of the xenon atom calculated using the regularized Lagrange-Laguerre mesh [26] is shown in Fig. 11. (Note that the large quantum defects of the low- $l$ states of Xe shift states with $n=10$ or 11 into the $\nu=7$ region.)

Figure 11 is essentially the same as the Stark map shown in Ref. [16], except that the naming of the states has been changed (since both directions of the applied field are considered here, we cannot assign specific vacuum or surface orientation to the Stark states). At zero field, states with $l$ $<4$ have nonzero quantum defects and are separated from the rest of the manifold; these states have been labeled with their associated $l$ quantum number at zero field. States with $l \geqslant 4$, which remain degenerate in energy at zero field, have been labeled $H 7$ for the highest-energy state within the manifold at nonzero field, $H^{\prime} 7$ for the second highest-energy state, and $L 7$ for the lowest-energy state. Unlike the case of a hydrogen atom, avoided level crossings occur for nonhydrogenic systems even in a homogeneous electric field (the Runge-Lenz vector [43] is no longer conserved).

\section{Wave-packet results for ion-extraction fields}

Figure 12 shows the results from our implementation of the wave-packet approach (using a CWDVR) for the $7 f, H 7$, and the $11 p$ states of xenon (solid lines) compared with the results obtained in the study by Sjakste et al. [16] (dashed lines) for a collisional velocity of $5 \times 10^{-3}$ a.u. and an ion- 


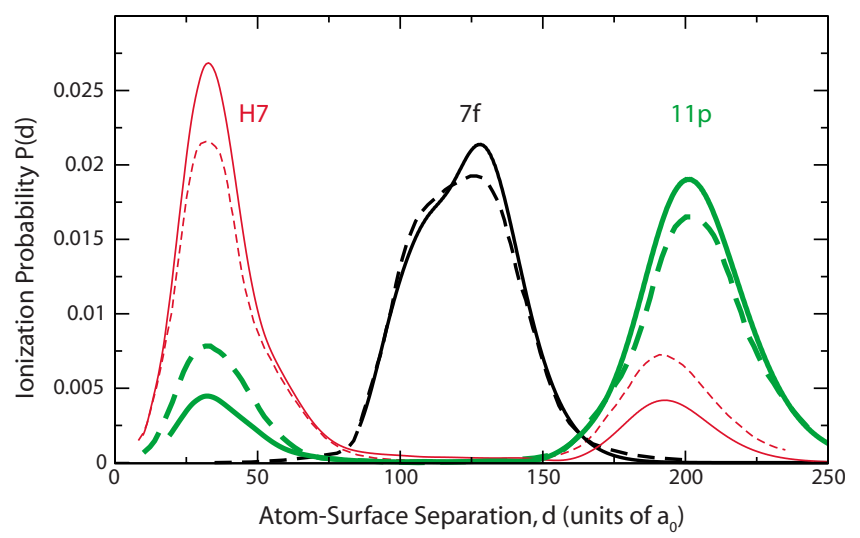

FIG. 12. (Color online) Wave-packet results for the $7 f, H 7$, and the $11 p$ states of xenon in an ion-extraction field of $F=1.6$ $\times 10^{-5}$ a.u. and a collisional velocity of $5 \times 10^{-3}$ a.u. The electron flux into the metal surface was measured by the forward $(\theta$ $>90^{\circ}$ ) radial flux plane. Solids lines are results obtained using the CWDVR described in this work, and dashed lines are the results of Sjakste et al. [16].

extraction field of $F=1.6 \times 10^{-5}$ a.u. $\left(\equiv 8.23 \times 10^{6} \mathrm{~V} \mathrm{~m}^{-1}\right)$ (this field value is close to the avoided crossing of the $\mathrm{H7}$ and $11 p$ states in Fig. 11.) Minor differences are observed in the relative magnitudes of the peaks of the two sets of results, which may be explained by the slightly different values of the parameters used in the pseudopotential.

The calculations using the "change of variables" method in Ref. [16] typically required $2000 \times 425$ grid points, whilst the calculations using the CWDVR outlined above require only $474 \times 70$ (radial $\times$ angular) points to produce the data presented in Fig. 12. This significant decrease in the number of grid points clearly shows that the CWDVR is better suited for ionization problems involving the Coulomb potential (such as the surface-ionization problem studied here) than a grid obtained from the coordinate transformations of uniformly distributed grids [16]. The reduction of the number of grid points decreases the number of operations per time step, and therefore allows for the possibility of carrying out calculations involving lower collisional velocities and/or Rydberg states of higher principal quantum numbers.

As explained in Ref. [16], the double peaks in the electron flux profiles shown in Fig. 12 for the $H 7$ and the $11 p$ states are attributed to the mixing of the "initially" surface-oriented [at large atom-surface separations $(d>350$ a.u. $)] 11 p$ state with the initially vacuum-oriented $H 7$ state as the xenon atom passes through an avoided crossing between the $H 7$ and $11 p$ states at a distance of $\approx 250 a_{0}$ from the surface (see Fig. 3 in Ref. [16]). A shoulder is observed in the surfaceoriented $7 f$ state curve in Fig. 12 at low atom-surface separation even though this state appears far in energy from other Rydberg states in the Stark map; this is due to an avoided crossing with the $L 7$ state at $\approx 130 a_{0}$ from the surface.

\section{Wave-packet results for electron-extraction fields}

Figure 13 shows the ionization dynamics of xenon Rydberg atoms in the presence of an electron-extraction field of $F=-1.4 \times 10^{-5}$ a.u. $\left(\equiv 3.06 \times 10^{3} \mathrm{~m} \mathrm{~s}^{-1}\right)$ for three different

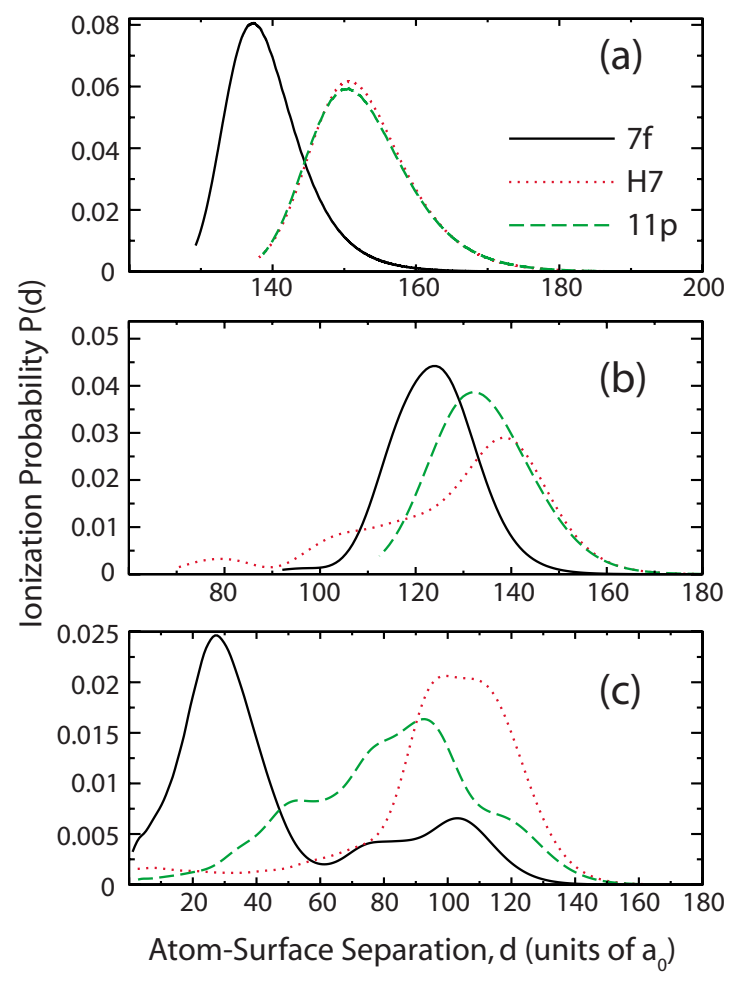

FIG. 13. (Color online) Wave-packet propagation results for the ionization of the $7 f, H 7$, and $11 p$ xenon Rydberg states approaching a metal surface in the presence of an electron-extraction field of $F=-1.4 \times 10^{-5}$ a.u. and with the collisional velocity of (a) 1.4 $\times 10^{-4}$ a.u., (b) $1.4 \times 10^{-3}$ a.u., and (c) $1.4 \times 10^{-2}$ a.u. Note the different scale of the $x$ axis on the three plots.

collisional velocities. Figure 14 shows the energies of the Rydberg states as they approach the metal surface at this field. At large distances all the Stark states in the $\nu=7$ energy range (except for the $11 \mathrm{~s}$ and $L 7$ states) are well spread apart in energy (see Fig. 11) but the $L 7$ to $H 7$ Rydberg states encounter numerous avoided crossings as they approach the metal surface. As in the case of the $\mathrm{H}$ atom (and for the same reason) there is an increased number of avoided crossings in the reversed electron-extraction field compared with the ion-

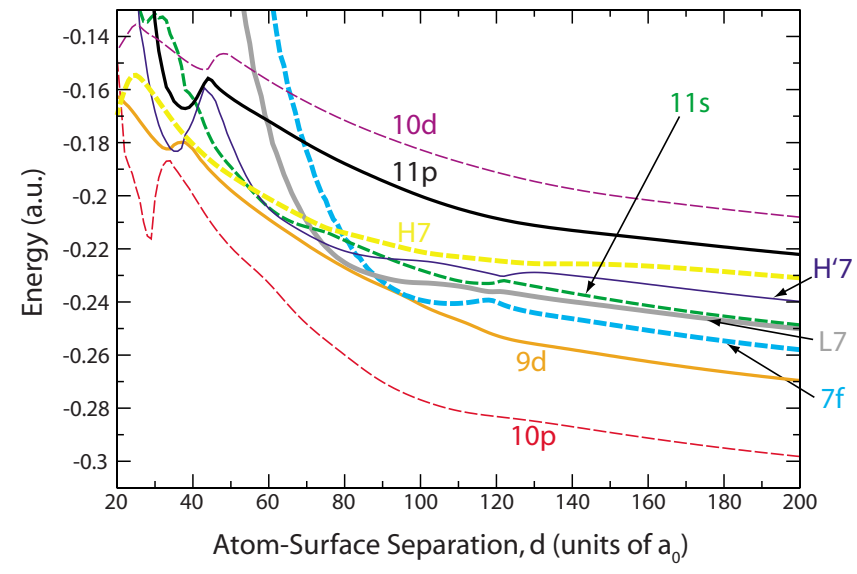

FIG. 14. (Color online) The energies of the xenon $n=7$ manifold in the presence of an electron-extraction field of $-1.4 \times 10^{-5}$ a.u. as a function of atom-surface separation. 
extraction field. The interactions between the Rydberg states are much stronger for the nonhydrogenic system compared with the hydrogenic system. In the case of hydrogen, level interactions only occur as a result of the perturbing surface potential. In contrast, for a nonhydrogenic atom, level interactions occur as a consequence of the externally applied electric field as well as the surface potential. The energy gap at the avoided crossings will generally be larger for Xe, making it more likely that each crossing is traversed more adiabatically. There are now more crossings however, and there is also an increased probability of level transition through nonadiabatic interactions between curves that are well separated, and which would be regarded as noncrossing. Thus, the ionization dynamics of the nonhydrogenic system are strongly dependent on the collisional velocity.

The wave-packet results for the $\nu=7$ Rydberg states at the lowest velocity [Fig. 13(a)] are very similar to those obtained from complex-scaling calculations in the adiabatic levelcrossing limit. Surface ionization occurs at relatively large distances before energy-level crossings occur and the process is mostly adiabatic. In contrast, the comparison of wavepacket results at the intermediate velocity [Fig. 13(b)] with complex-scaling calculations shows that there is a significant nonadiabatic transfer of population amongst the manifold, and the ionization dynamics of the Rydberg states cannot be described correctly using the time-independent approach.

For the lowest and intermediate velocity chosen in these calculations [Figs. 13(a) and 13(b)], ionization of all three states occurs principally at quite a similar distance. As shown in Fig. 14, the vacuum-oriented $11 p$ state does not share an avoided crossing with its energetically adjacent states at separations $>50 a_{0}$, accounting for its single-peaked ionization curve (i.e., it is fully ionized before passing any avoided crossings). Figure 15 shows that the electronic wave function of the $11 p$ state is much less polarized in the field than that of the $H 7$ state, which has contributions from zero-field states with higher angular momentum. Although the $11 p$ state (which in the presence of a field, does not have pure $p$ character) is mostly vacuum oriented, there is still a significant fraction of its total wave function on the surface side of the ion core, with an extension towards the surface similar to that of the $H 7$ state. As a result, the onset of ionization for the $11 p$ and $H 7$ states is very similar in the presence of the electron-extraction field. This can be contrasted with the case of the ion-extraction field, where the $11 p$ and $H 7$ states ionize at very different separations from the surface (see the peak positions in Fig. 12). In the ion-extraction field, the $H 7$ state is far more polarized towards the vacuum than the $11 p$ state in the electron-extraction field (imagine that the surface is located in the positive- $z$ direction in Fig. 15 instead), and so it will ionize much closer to the surface.

At the highest calculated velocity [Fig. 13(c)] all states show a highly complex behavior. The avoided crossings encountered as the Rydberg states approach the metal surface are crossed more diabatically than at lower velocities, and thus retain more of their ionization characteristics from the wave function at large distances from the surface, i.e., the largest ionization peak of the vacuum-oriented (when far from the surface) $7 f$ and $11 p$ states appears much later than the surface-oriented $H 7$ state compared with the lower ve-

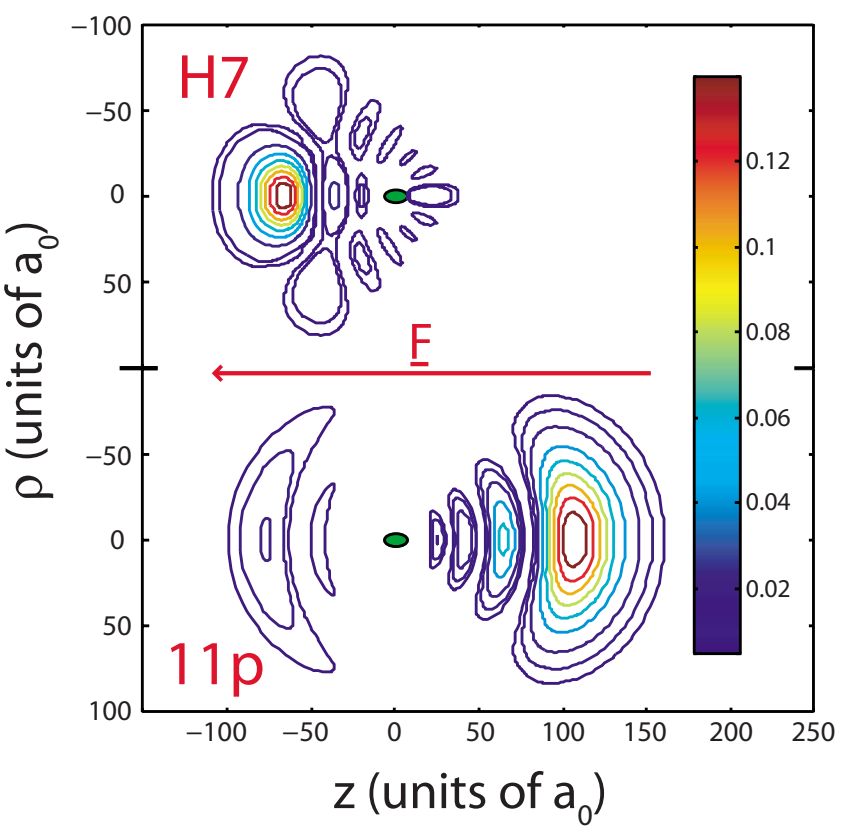

FIG. 15. (Color online) Plot of the electronic wave function of the $H 7$ and $11 p$ states in the presence of an electron-extraction field of $F=-1.4 \times 10^{-5}$ a.u. The metal surface is located at $z=-380 a_{0}$.

locities. However, even at a collisional velocity of 1.4 $\times 10^{-2}$ a.u. there are still significant adiabatic contributions from other Rydberg states, which can be seen by the multiple-peaked structures of the ionization curves.

\section{Backscattering of the electron flux}

For the hydrogenic case, it was shown that when the electron-extraction field is just below that required for field ionization, the most vacuum-oriented states are "surface" ionized by losing the electron toward the vacuum side of the ion core. For the xenon atom, however, the backscattering of the Rydberg electron can occur for any state that mixes with a vacuum-oriented state as it approaches the surface.

Figure 16 shows the fraction of forward-scattered electron flux and backscattered electron flux for the various higherenergy states of the $\nu=7$ xenon Rydberg manifold at an electron-extraction field of $F=-2.3 \times 10^{-5}$ a.u. In the presence of this externally applied field, the $11 p$ and $H^{\prime} 7$ states are vacuum oriented, and the $H 7$ state is surface oriented at large distances from the surface. Although it is predominately the vacuum-oriented states that exhibit a backscattered electron flux, the mixing amongst the three states as they approach the surface (shown by the shared ionization peaks) results in the $H 7$ state gaining some vacuum-oriented characteristics on its trajectory towards the surface, and consequently some of the electron density is backscattered.

\section{Summary of the xenon atom results}

It is shown that for xenon, as in the case of hydrogen, the reversed orientation of the wavefunctions with respect to the surface in the reversed electron-extraction field can have a profound effect on the relative dynamics of the Rydberg states. Due to the stronger level interactions in the nonhydro- 


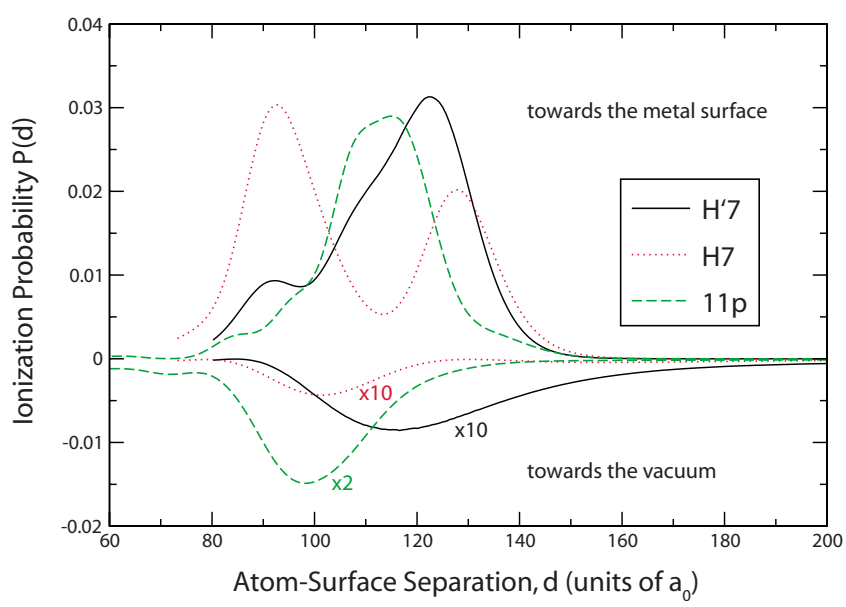

FIG. 16. (Color online) The electron flux measured by the forward $\left(\theta>90^{\circ}\right)$ and backward $\left(\theta<90^{\circ}\right)$ radial flux planes (positioned at $\left.r_{\mathrm{abs}}=345 a_{0}\right)$ plotted as a function of atom-surface separation for the $H^{\prime} 7, H 7$, and $11 p$ xenon Rydberg states colliding with the surface with the collisional velocity of $1.4 \times 10^{-3}$ a.u. and in the presence of an electron-extraction field of $F=-2.3 \times 10^{-5}$ a.u. The flux measured by the backward flux plane is plotted on the negative axis.

genic system, the ionization dynamics are affected by more complex nonadiabatic transitions, which can only be properly described by time-dependent calculations. At sufficiently high electron-extraction fields (just before direct-field ionization), the ionization dynamics is further complicated by the possibility of backscattered ionization for states that exhibit or gain vacuum-oriented characteristics (through coupling with other close-in-energy Rydberg states) as they approach the metal surface. Overall the differences between the ionand electron-extraction fields are even more pronounced for the xenon Rydberg atom than the hydrogen atom.

\section{Constant collisional velocity approximation}

It is important to note that the results presented in this work have been calculated under the assumption that the ion core is moving at a constant velocity (the initial collisional velocity). We have applied this constant velocity assumption as a first approximation to allow a comparison of our numerical results with the work of Sjakste et al. [16], and to demonstrate that our application of the Coulomb-wave DVR can potentially produce theoretical results at the experimental range of principal quantum numbers and velocities. We have also used this simple approximation to examine the qualitative effects of changing the direction of the applied field, and the dependence of nonadiabatic effects on the velocity of the ion core. However, the constant-velocity approximation cannot be correctly justified at the low collisional velocities (in some cases comparable to experimental velocities $[4,5])$ that are studied in this paper. At such low velocities, the trajectory of ion core is susceptible to the forces from the Rydberg electron and its image charge, and the image charge of the ion core.

To correctly describe the dynamics of the Rydberg electron and the ion core, the coupling between the two systems

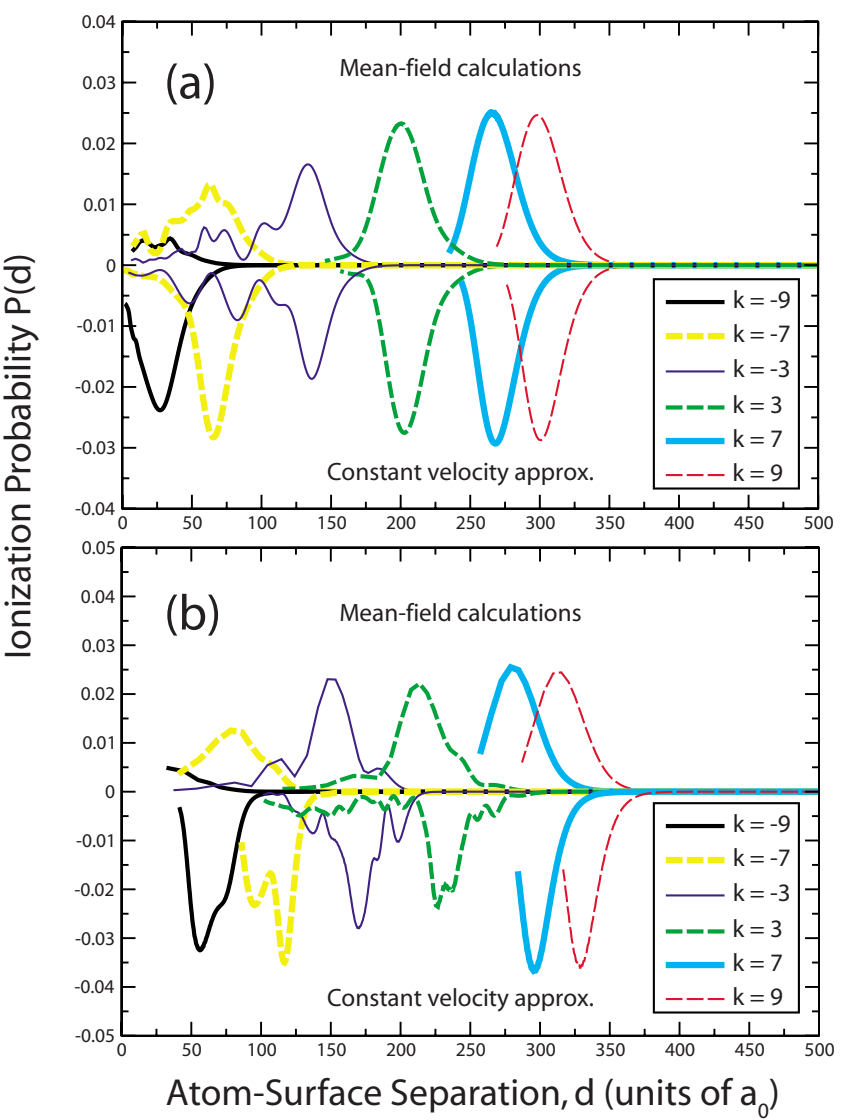

FIG. 17. (Color online) Comparison of the wave-packet results for various $n=10$ hydrogen atom Rydberg states obtained using the constant velocity approximation (negative axis) with results obtained with the ion core moving under the mean field of the Rydberg electron and its image charge (positive axis). The magnitude of the extraction field is $F=-1 \times 10^{-6}$ a.u., and the initial collisional velocities are (a) $1.4 \times 10^{-3}$ a.u. and (b) $1.4 \times 10^{-4}$ a.u.

must be correctly included. We have carried out preliminary mean-field calculations [44] to evaluate the validity of the constant velocity approximation. The expectation value of the electronic Hamiltonian (i.e., the mean-field potential) is calculated "on the fly," and the motion of the ion core under this mean field (which also includes the ion-core-image-ioncore attraction) is treated classically and propagated using a velocity Verlet algorithm. The electronic dynamics is treated quantum mechanically as before. In these preliminary calculations (carried out in the atomic-core centered frame), we have not included the electronic phase effects due to the accelerating moving frame.

The calculations show that the mean-field potential results in an overall acceleration of the ion core as the surface is approached. This acceleration generally increases closer to the surface as it becomes more dominated by the ion-imageion attraction. The increasing velocity of the ion core reinforces the need for a time-dependent wave-packet-type approach to capture some of the nonadiabatic effects occurring during ionization. Figure 17 presents a comparison of the results of the mean-field calculation with the constantvelocity calculations for various hydrogen atom Rydberg states at the field of $F=-1 \times 10^{-6}$ a.u., and the initial colli- 
sional velocity of $1.4 \times 10^{-3}$ a.u. and $1.4 \times 10^{-4}$ a.u.

The main effects of the increase of collisional velocity as the Rydberg atom approaches the surface are to shift the onset of ionization, and to extend the "tail" of the ionization curves towards lower atom-surface separations. As expected, these effects are much more pronounced for the lower initial collisional velocity. Since the acceleration of the Rydberg atom increases with smaller atom-surface separations, Rydberg states that ionize closest to the surface are most strongly affected. For the latest ionized, most vacuum-oriented states shown in Fig. 17, the final collisional velocities are up to an order of magnitude greater than the initial velocity. This increase in collisional velocity increases the extent of nonadiabatic transitions, as shown by the range of constant collisional velocity studies presented in this work. These types of changes in the ionization curves, caused by the acceleration of the ion core, are much smaller for the heavier xenon atom system.

Although at the low-collisional velocities, the wavepacket propagation results for the mean-field calculations show significant differences from the constant velocity approximation calculations, the qualitative findings on the effects of changing the direction of the applied field, and the dependence of nonadiabaticity on the velocity of the ion core given in this work are general and remain valid for both accelerating and constant-velocity systems. However, it appears that in order to quantitatively compare the calculations with the experimental results, inclusion of ion-acceleration effects will be required. Application of the mean-field approach to reproduce the experimental results will be the focus of our future theoretical work.

\section{CONCLUSIONS}

In this paper, we have developed a wave-packet propagation approach involving the Coulomb-wave DVR (CWDVR) to treat the surface ionization of a Rydberg atom. By simply varying the two grid parameters that control the distribution of grid points at short and long range, the CWDVR can efficiently treat both the bound electronic wave function close to the ion core and the free electron wave, which has escaped the Coulomb attraction. With this CWDVR, we have demonstrated that the wave-packet propagation calculations for the surface ionization of Rydberg atoms can be carried out more efficiently (with fewer grid points) than with the grids employed previously [16], which should allow us to produce quantitative results at the experimental range of principal quantum numbers in the future.

The time-dependent wave-packet propagation approach and the time-independent complex-scaling approach are two complementary methods for studying the surface-ionization problem. The complex-scaling approach can provide information on which Rydberg states are interacting with the selected state of interest and on the positions of their avoided crossings as the Rydberg atom approaches the metal surface. The calculated widths and lifetimes provide information on the ionization dynamics of the Rydberg state in the adiabatic limit. Through comparison with the wave-packet results, which include all nonadiabatic effects, the extent of nonadia- batic transitions that occur in the real dynamical system at the given collisional velocity can be analyzed.

Using the wave-packet propagation approach involving the CWDVR, alongside the complex-scaling approach, we have focused on the effects of the direction of the externally applied electric field (ion- and electron-extraction fields), on the surface-ionization dynamics of the $\mathrm{H}$ and $\mathrm{Xe}$ atoms. It is found that for both systems, the ionization dynamics are very different for the ion- and the electron-extraction schemes. In the negative electron-extraction fields, the orientation of the Rydberg electron density with respect to the surface is reversed and the states are likely to encounter more avoided crossings as the atom approaches the metal surface. Consequently, the wave function may switch character more readily, but careful attention needs to be paid to the adiabatic versus nonadiabatic nature of passage through these avoided crossings. The ionization curves (ionization probability versus distance plots) are therefore strongly dependent on the collisional velocity, and are often found to exhibit complicated oscillations over a wide range of atom-surface separations. For a small range of fields just before direct-field ionization takes place, an additional ionization mechanism is predicted for the Rydberg atom in the presence of an electron-extraction field. For the most vacuum-oriented states, as the atom approaches the surface, a significant fraction of the Rydberg electron density can escape towards the vacuum side of the ion core (the electron is backscattered with respect to the nuclear trajectory). Rydberg states that mix with vacuum-oriented states may also exhibit backscattering, particularly for the strongly interacting nonhydrogenic system. For the ion-extraction scheme, the fraction of backscattered electron density is negligible.

The work presented in this paper has been motivated in part by the recent experiments involving the interaction of $\mathrm{H}_{2}$ Rydberg molecules with metal surfaces [21], where we have succeeded in detecting an electron signal from the surface ionization by applying an electron-extraction field. The electron signal is observed over a considerably wider range of fields and states than is predicted here for the atomic systems. The molecular system, however, introduces an additional level of complexity in that the rotational degrees of freedom of the ion core are found to contribute to the surface ionization process. An exchange of energy between the rotation and the Rydberg electron occurs, enhancing the surface ionization in a phenomenon we described previously as "surface-induced autoionization" $[4,5]$. A much greater number of level crossings can also occur. The effect of the rotation-electronic energy exchange is to allow surface ionization at considerably greater distances than would be expected for comparable atomic systems, and this may allow backscattering of electron flux to occur more readily. The increased number of level crossings will also allow every state to develop vacuum-oriented character at relatively low fields. The present calculations do not address the rotational coupling with the Rydberg electron and therefore cannot be compared directly with experimental measurements of electron-extraction probabilities; the inclusion of this additional degree of freedom is the subject of ongoing work in our group.

In the future it may be of experimental interest to study the surface ionization in the presence of an electron- 
extraction field for systems of greater chemical complexity: in the negative field configuration the ion core is directed towards (instead of being pulled away from) the metal surface, allowing subsequent low-energy ion-surface collision processes to occur. These processes might include bond dissociation (for a molecular ion), neutralization (by the metal surface or adsorbates) followed by bond dissociation or bond formation (with adsorbates) and other ion-surface chemistry. To determine the collisional energy of the ion at the surface where these processes can occur, the distance at which the surface ionization occurs needs to be known (i.e., the variation of the probability of surface ionization with atom- surface separation); this is given directly by the results of wave-packet propagation studies such as those carried out in this work.

\section{ACKNOWLEDGMENTS}

The authors are grateful to the EPSRC for their financial support of this work, and to the Oxford Supercomputing Centre for their computing resources. We also thank Professor D. E. Manolopoulos for his help with the wave-packet propagation programs.
[1] C. Fabre, M. Gross, J. M. Raimond, and S. Haroche, J. Phys. B 16, L671 (1983).

[2] D. F. Gray, Z. Zheng, K. A. Smith, and F. B. Dunning, Phys. Rev. A 38, 1601 (1988).

[3] C. A. Kocher and C. R. Taylor, Phys. Lett. A 124, 68 (1987).

[4] G. R. Lloyd, S. R. Procter, and T. P. Softley, Phys. Rev. Lett. 95, 133202 (2005).

[5] G. R. Lloyd, S. R. Procter, E. A. McCormack, and T. P. Softley, J. Chem. Phys. 126, 184702 (2007).

[6] S. B. Hill, C. B. Haich, Z. Zhou, P. Nordlander, and F. B. Dunning, Phys. Rev. Lett. 85, 5444 (2000).

[7] F. B. Dunning, S. Wethekam, H. R. Dunham, and J. C. Lancaster, Nucl. Instrum. Methods Phys. Res. B 258, 61 (2007).

[8] P. Nordlander and J. C. Tully, Surf. Sci. 211, 207 (1989).

[9] J. Hanssen, C. F. Martin, and P. Nordlander, Surf. Sci. 423, L271 (1999).

[10] K. Ganesan and K. T. Taylor, J. Phys. B 29, 1293 (1996).

[11] A. G. Borisov, D. Teillet-Billy, and J. P. Gauyacq, Nucl. Instrum. Methods Phys. Res. B 78, 49 (1993).

[12] A. G. Borisov, R. Zimny, D. Teillet-Billy, and J. P. Gauyacq, Phys. Rev. A 53, 2457 (1996).

[13] D. Wang, M. L. Du, and S. Lin, J. Phys. B 39, 3529 (2006).

[14] F. B. Dunning, H. R. Dunham, C. Oubre, and P. Nordlander, Nucl. Instrum. Methods Phys. Res. B 203, 69 (2003).

[15] A. K. Kazansky, A. G. Borisov, and J. P. Gauyacq, Nucl. Instrum. Methods Phys. Res. B 157, 21 (1999).

[16] J. Sjakste, A. G. Borisov, and J. P. Gauyacq, Phys. Rev. A 73, 042903 (2006).

[17] B. Bahrim and U. Thumm, Surf. Sci. 521, 84 (2002).

[18] J. Burgdörfer, P. Lerner, and F. W. Meyer, Phys. Rev. A 44, 5674 (1991).

[19] N. Shenvi, H. Cheng, and J. C. Tully, Phys. Rev. A 74, 062902 (2006).

[20] N. Lorente, D. Teillet-Billy, and J.-P. Gauyacq, J. Chem. Phys. 111, 7075 (1999).

[21] E. A. McCormack, M. S. Ford, and T. P. Softley (unpublished).

[22] A. R. Canário, A. G. Borisov, J. P. Gauyacq, and V. A. Esau- lov, Phys. Rev. B 71, 121401(R) (2005).

[23] K. M. Dunseath, J.-M. Launay, M. Terao-Dunseath, and L. Mouret, J. Phys. B 35, 3539 (2002).

[24] L. Mouret, K. M. Dunseath, M. Terao-Dunseath, and J.-M. Launay, J. Phys. B 36, L39 (2003).

[25] L.-Y. Peng and A. F. Starace, J. Chem. Phys. 125, 154311 (2006).

[26] D. Baye, Phys. Status Solidi B 243, 1095 (2006).

[27] J. Los and J. J. C. Geerlings, Phys. Rep. 190, 133 (1990).

[28] P. J. Jennings, R. O. Jones, and M. Weinert, Phys. Rev. B 37, 6113 (1988).

[29] J. C. Light and T. Carrington, Jr., Adv. Chem. Phys. 114, 263 (2000).

[30] A. R. Barnett, Comput. Phys. Commun. 27, 147 (1982).

[31] W. H. Press, S. A. Teukolsky, B. P. Flannery, and W. T. Vetterling, Numerical Recipes in FORTRAN: The Art of Scientific Computing (Cambridge University Press, New York, NY, 1992).

[32] M. D. Feit and J. A. Fleck, Jr., J. Chem. Phys. 78, 301 (1983).

[33] A. M. Cohen, Linear Algebr. Appl. 240, 183 (1996).

[34] D. E. Manolopoulos, J. Chem. Phys. 117, 9552 (2002).

[35] T. Gonzalez-Lezana, E. J. Rackham, and D. E. Manolopoulos, J. Chem. Phys. 120, 2247 (2004).

[36] T. Seideman and W. H. Miller, J. Chem. Phys. 96, 4412 (1992).

[37] C. E. Moore, Atomic Energy Levels Natl. Bur. Stand. (U.S.) (U. S. GPO, Washington, D.C., 1958), Vol. III.

[38] L. G. Wang and R. D. Knight, Phys. Rev. A 34, 3902 (1986).

[39] J. N. Bardsley, Case Stud. At. Phys. 4, 299 (1974).

[40] H. Suno, L. Andric, T. P. Grozdanov, and R. McCarroll, Eur. Phys. J. D 13, 213 (2001).

[41] T. F. Gallagher, Rydberg Atoms (Cambridge University Press, Cambridge, UK, 1994).

[42] J. R. Rubbmark, M. M. Kash, M. G. Littman, and D. Kleppner, Phys. Rev. A 23, 3107 (1981).

[43] P. J. Redmond, Phys. Rev. 133, B1352 (1964).

[44] J. C. Tully, Faraday Discuss. 110, 407 (1998). 